\title{
Superficial pemphigus
}

INSERM

\section{Source}

INSERM. (1999). Orphanet: an online rare disease and orphan drug data base. Superficial pemphigus. ORPHA:46485

Superficial pemphigus is a rare, autoimmune, bullous skin disease characterized clinically by multiple flaccid blisters, typically occurring on the face, scalp, trunk and extremities, which rapidly evolve into scaly, crusted, pruritic skin erosions. Histopathologically, superficial acantolytic blisters, as well as intercellular deposits of IgG autoantibodies directed against desmoglein 1 (and occasionally against desmoglein 3) in the upper epidermis, are observed. 